\title{
RÉFLEXIONS CONCERNANT LES PROBLÈMES DFCI DES ESPACES FORESTIERS LANDAIS À LA LUMIËRE DES INCENDIES DU PORGE - LACANAU
}

NDLR

L'article qui suit s'articule - hélas! - en deux textes complémentaires: le premier — "Réflexions concernant ..." - avait été rédigé, puis envoyé à notre Revue avant le 30 mars 1990.

II avait, malheureusement, valeur prémonitoire, puisqu'il dut être complété, le 6 juillet 1990, par un deuxième texte intitulé "Les incendies du 31 mars 1990 en Gironde".

\section{LES INCENDIES DU PORGE - LACANAU, 1989}

Le mardi 18 juillet 1989 , le $1362^{e}$ incendie de forêts, que connaît le département de la Gironde depuis le début d'une année exceptionnelle, démarre quelque part entre les communes du Porge et de Lacanau au lieu-dit "Lande de Mistre ".

Au total, les 426 pompiers professionnels de la DFCl girondine auront 2336 feux à combattre sur l'annèe 1989 pour une superficie forestière incendiée de 5000 ha.

L'incendie du Porge-Lacanau, qui aura mobilisé jusqu'à 2000 hommes et progressé durant les journées du 18,19 et 20 juillet, sera pour sa part responsable de la destruction de 3700 ha de forêt de Pin maritime.

II sera accompagné de l'évacuation de plus de 5000 personnes, touristes des campings côtiers essentiellement.

Enfin, il aura nécessité, pour être maîtrisé, l'engagement de cinq Canadair arrivés de Marignane à partir du jeudi matin, 20 juillet.

Présenté de manière aussi laconique, il offre à l'évidence des caractéristiques si inhabituelles à

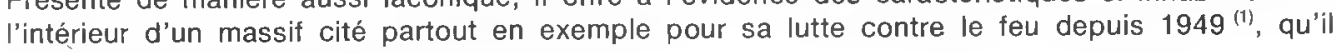
mérite une analyse particulière.

C'est qu'en effet, en ces journées exceptionnellement chaudes, bien des certitudes érigées en idées reçues ont vacillé ; quant aux lancinantes polémiques sur le financement de la DFCl dans les Landes et en Lot-et-Garonne, c'est peu dire qu'elles ont paru bien mesquines eu égard aux enjeux économiques et écologiques mis en évidence.

(1) 47000 ha dètruits durant le mois d'août 1949 et surtout 82 victimes dans le seul dèpartement de la Gironde. 


\section{BREFS RAPPELS DES FAITS}

\section{- Mardi 18 juillet}

Entre $16 \mathrm{~h} 30$ et $23 \mathrm{~h}$, on dénombrera près de 200 pompiers mobilisés contre l'incendie attisé par un vent tourbillonnant rendant la lutte difficile. Dès que le feu prendra une certaine ampleur, tous les témoins observeront des flottements dans l'organisation; des renforts sont sollicités auprès des départements voisins. Les premières évacuations de colons et de campeurs ont lieu à l'initiative du Sous-Préfet De Lesparre.

- Nuit du 18 au 19 juillet

Le vent s'étant calmé, le feu paraît maîtrisé vers deux heures du matin. À ce moment-là, plusieurs unités regagnent leur centre, l'ampleur inhabituelle du sinistre empêche les maigres effectifs restants de mettre en œuvre les précautions élémentaires en pareil cas : circonscription de la zone par une bande de $10 \mathrm{~m}$ environ traitée à sable blanc et arrosée afin d'éviter les nouveaux départs de feu. La sous-estimation du risque cette nuit-là demeure pour beaucoup de spécialistes une erreur lourde de conséquence.

- Mercredi 19 juillet

À partir de midi, on note des reprises de feu multiples attisées par un vent de sud-est assez fort qui n'arrive pas à tempérer une atmosphère étouffante. Le large canal qui relie l'étang de LèdeBasse à celui de Lacanau ne constitue pas un gros obstacle pour le feu qui va dès lors progresser vers l'ouest avec un front de deux kilomètres de large. L'arrivée du Préfet de Région sur les lieux et son installation au PC mobile n'entraînent pas immédiatement les clarifications nécessaires. L'avancée du feu dans les zones dunaires occupees par la forêt communale du Porge complique la tâche des sauveteurs en raison des difficultés de circulation des engins de lutte dans les sables pulvérulents. On évacue les 2800 personnes d'un camping puis la plage du Porge, enfin le camp naturiste de la Jenny. Ce jour-là, un Fokker 27 fait son apparition dans le ciel de Lacanau. Sa capacité à combattre le feu apparaîtra immédiatement comme très limitée. Au soir du 19 juillet, le feu a parcouru environ 1500 ha. Devant l'impossibilité d'acheminer des renforts de pompiers forestiers de départements voisins, requis par des incendies multiples, on

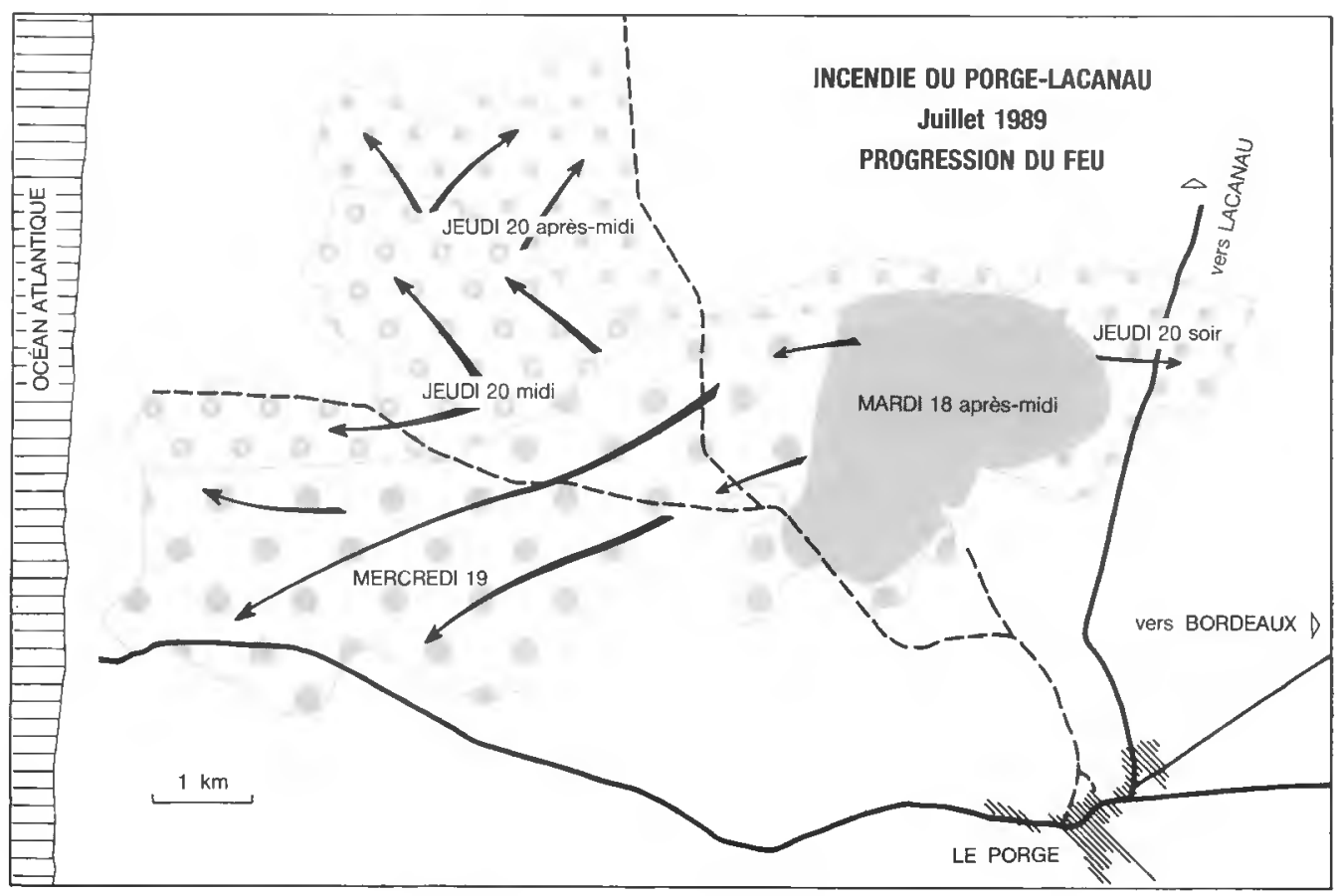


fait appel à l'armée pour évacuer les personnes et aider à la lutte active. Les sapeurs-pompiers de l'île-de-France sont appelés à la rescousse.

- Jeudi 20 juillet

L'engagement de tous les renforts (1 400 hommes), l'entrée en action de cinq Canadair et une meilleure coordination vont permettre une lutte plus efficace. Le feu progressant néanmoins vers le nord, on envisage un instant l'évacuation de Lacanau-Océan.

Dans la soirée de jeudi, alors qu'on note une accalmie sur le front Nord de l'incendie, le feu repart à l'est, dévorant une centaine d'hectares.

II sera stoppé en limite de la forêt communale de Saumos par l'action conjointe des Canadair et d'un contre-feu allumé à l'initiative du maire.

L'heure du bilan, des évaluations et des polémiques avait sonné.

\section{ÉVALUATION DES DÉGÂTS}

Les estimations conduites simultanément par le Centre de Productivité et d'Action forestière d'Aquitaine (CPFA) pour la forêt privée et l'Office national des Forêts (ONF) pour la forêt publique donnent les chiffres suivants:

- surface incendiée .................................. 3654 ha,

- volume des bois commercialisables .................... environ $300000 \mathrm{~m}^{3}$,

- perte de valeur d'avenir .......................... environ $30 \mathrm{MF}$.

Répartition par classe d'âge

\begin{tabular}{|c|c|}
\hline \multicolumn{2}{|c|}{$\begin{array}{c}\text { Forêt privée } \\
\text { (107 propriétaires) }\end{array}$} \\
\hline Nature et âge & $\begin{array}{l}\text { Surfaces } \\
\text { (ha) }\end{array}$ \\
\hline Taillis . . . . . . . . . . & 10 \\
\hline Marais . . . . . . . . . . & 58 \\
\hline Coupes rases . . . . . . & 133 \\
\hline $0-4$ ans $\ldots . . .$. & 89 \\
\hline $5-9$ ans $\ldots . . .$. & 417 \\
\hline $10-14$ ans...... & 99 \\
\hline $15-19$ ans $\ldots \ldots \ldots$ & 194 \\
\hline $20-24$ ans...... & 287 \\
\hline $25-29$ ans...... & 236 \\
\hline $30-34$ ans...$\ldots$. & 244 \\
\hline $35-39$ ans $\ldots . . .$. & 217 \\
\hline $40-44$ ans $\ldots . . .$. & 69 \\
\hline $45-49$ ans $\ldots \ldots \ldots$. & 39 \\
\hline $50-54$ ans $\ldots \ldots \ldots$ & 9 \\
\hline 55 et $+\ldots \ldots \ldots$ & 133 \\
\hline TOTAL $\ldots \ldots \ldots \ldots$ & 2234 \\
\hline
\end{tabular}

\begin{tabular}{|r|c|c|c|c|}
\hline \multicolumn{5}{|c|}{ Forêt soumise au régime forestier } \\
\hline Type de peuplement & $\begin{array}{c}\text { FD } \\
\text { Lacanau }\end{array}$ & $\begin{array}{c}\text { FD } \\
\text { Porge }\end{array}$ & $\begin{array}{c}\text { FC } \\
\text { Porge }\end{array}$ & Total \\
\hline Semis $0 / 5$ ans.... & & & 190 & 190 \\
$6 / 12$ ans.... & & & 324 & 324 \\
$13 / 20$ ans .... & & & 116 & 116 \\
$21 / 35$ ans.... & 140 & 25 & - & 165 \\
35 ans ..... & 167 & 16 & 12 & 194 \\
\hline TOTAL ......... & 307 & 40 & 640 & 990 \\
\hline
\end{tabular}

\section{ACTIONS POST-INCENDIE}

Le moment de stupeur et parfois d'incrédulité passé, de très nombreuses initiatives ont vu le jour. Si les actions conduites par les partenaires traditionnels de la filière-bois (sylviculteurs, exploitants forestiers, administration forestière, ONF, associations de DFCl) sont demeurées 
relativement classiques, on doit souligner deux faits nouveaux qui méritent réflexion :

- l'engagement des collectivités locales dans le débat,

- la progressive médiatisation de l'incendie et les tentatives de récupération qui en résultent.

\section{Les acteurs de la filière-bois}

Les propriétaires sinistrés tout d'abord n'ont pas tardé à se regrouper en "Association des sinistrés de l'incendie de juillet 1989 ", ce qui leur a permis, avec le soutien du Syndicat des Sylviculteurs du Sud-Ouest, de mener des négociations multiples notamment avec les pouvoirs publics et les représentants du Fonds forestier national (FFN). Les discussions préalables aux ventes de bois se sont faites en revanche de gré à gré. Pour I'ONF, la commercialisation a eu lieu par vente publique.

D'une manière générale, on peut dire que la vente des bois d'œuvre s'est déroulée dans d'assez bonnes conditions et que les bois de trituration ont trouvé preneur rapidement même si leur exploitation a été différée dans le temps.

Parallèlement, I'ONF, la Maison de la Forêt (via le CPFA) et I'INRA prenaient l'initiative d'une mission aérienne qui, effectuée le 2 août, permettrait de détenir une photographie de l'état des peuplements avant que les exploitations ne commencent et de bâtir une cartographie de synthèse après observation au sol.

Enfin, la Fédération girondine des Associations syndicales de DFCl réunissait les principaux acteurs de la lutte afin de recueillir le maximum de témoignages.

Au total donc, une série d'actions qui démontraient in fine la volonté de réaction des acteurs traditionnels de la filière-bois face à un tel sinistre.

\section{Les collectivités publiques}

Sans méconnaître la capacité de l'État (via le FFN) à mettre d'importants crédits de reboisement à la disposition des sinistrés, sans passer sous silence la valse hésitation concernant la forêt non soumise (épineux problème), on est frappé d'assister à l'implication directe du Conseil régional puis du Conseil général par une série de mesures financières destinées à atténuer les conséquences du sinistre. II s'agit, bien sûr, des conséquences de la loi sur la décentralisation qui tend à mettre les collectivités locales en première ligne. Encore faut-il noter que, si le Conseil général se trouve très impliqué dans la lutte en raison de son effort de financement du budget des sapeurs-pompiers, le Conseil régional, lui, ne participe pas à l'action de DFCl en Aquitaine. Aussi, le million de francs qu'il met immédiatement à la disposition des sinistrés semble ressortir d'un "effet-annonce" plutôt que d'une réelle volonté de s'impliquer.

Dès lors, le Conseil général sera conduit, lui aussi, à prendre des mesures d'accompagnement concernant des renoncements à perception de taxe foncière sur une superficie double de la zone sinistrée et à diligenter une nouvelle étude concernant l'équipement de celle-ci.

La " société civile"

Ce qui, à notre sens, confère à l'incendie du Porge-Lacanau une spécificité historique déterminante, outre son ampleur, demeure la singulière efflorescence d'initiatives venues de ce que nous pourrions nommer "la société civile" par opposition aux professionnels de la filière et aux politiques.

Tout se passe en effet comme si, progressivement, au cours de l'hiver 1989-90, une appropriation externe du phénomène s'opérait au travers de multiples intervenants. À cet égard, la presse régionale joue un rôle d'accompagnement puissant en offrant une tribune à tous ceux qui désirent se manifester, les uns en toute bonne foi, les autres avec une stratégie bien établie.

L'analyse des articles consacrés par le grand quotidien régional Sud-Ouest aux conséquences de l'incendie du Porge montre à l'évidence que professionnels et politiques sont peu à peu dépossédés de leur sujet par les intervenants extérieurs.

Sans viser à l'exhaustivité, citons rapidement quelques-unes des actions qui trouvent un large écho médiatique régional au cours de l'hiver 1989-90. 


\section{- Août 1989}

Création d'une association "Vive la Forêt" au Porge, en présence de 300 personnes. "Une association Vive la Forêt indépendante des mairies, des partis et sans but lucratif "qui veut "rassembler des personnes pour que cette forêt reste créatrice de richesses au service de la population, fermée à la spéculation immobilière et à une forme de tourisme inadapté à la forêt " (Sud-Ouest).

\section{- Octobre 1989}

La Société Aquitaine Informatique lance une randonnée en direction du Porge. Chaque participant se verra remettre un pin en venant retirer son dossard.

"Ainsi, il s'agira... non seulement d'afficher son attachement à une région belle et attirante cruellement éprouvée, mais aussi d'apporter personnellement la preuve que la qualité de la vie et la richesse d'une région, c'est bien l'affaire de tous et pas uniquement celle de quelques élus qui doivent se débattre dans le maquis des réglementations, des crédits, des aides et des soutiens officiels divers" (Sud-Ouest).

- Octobre 1989

Réunion au Porge de la Fédération nationale de la Forêt affiliée au Mouvement national de Lutte pour l'Environnement.

\section{- Décembre 1989}

La société "Miele" offre 1000 pins à la commune du Porge et crée une association baptisée "L'Écho Pins" destinée à recueillir les fonds provenant de la vente d'œuvres d'art qu'elle expose.

- Janvier 1990

3000 enfants, réunis par la Fédération nationale de l'Office central de Coopération à l'École, plantent un arbre sur les lieux du sinistre.

\section{- Février 1990}

Les élèves du collège d'horticulture de Creon plantent 120 Ginkgo biloba sur un pare-feu du Porge. "Un arbre extraordinaire qui sera désormais celui de la liberté sur la place du Porge" (Sud-Ouest, 7 février 1990).

Cette initiative trouvera un large écho médiatique dans la presse écrite et parlée. On pourrait bien sûr se livrer à une analyse approfondie de tous ces textes et montrer comment l'espace forestier, devenu partiellement mythique, devient un champ d'interventions multiples.

\section{QUESTIONS - ÉLÉMENTS DE RÉPONSE}

Demeurent des questions lancinantes qu'on ne peut s'empêcher de poser:

- Comment expliquer le développement d'un incendie aussi important, au sein d'un massif forestier équipé et cité en exemple pour l'efficacité dont il fait preuve depuis 1949 dans la lutte contre le feu?

- Quelles sont les leçons à en tirer et les propositions à avancer pour éviter le renouvellement de ce genre de sinistre?

\section{Éléments d'analyse}

"Paradoxalement, alors que le système landais fait annuellement la preuve de son efficacité, les partenaires de 1945 s'approchent, consciemment ou non, de l'heure qui verra l'implosion du système... II est à craindre malheureusement que seul un choc psychologique dans l'opinion publique, analogue à celui des "années noires" (1945 à 1949), ramènera tous les partenaires, les communes landaises au premier chef, à plus de raison" (José Manarillo, 1985).

Il existe des causes profondes à l'incendie du Porge-Lacanau: elles résultent, pour l'essentiel, d'une perte de la mémoire collective des ravages des incendies de forêts et d'une socialisation 
progressive du risque sous l'effet de la pénétration forestière par les voies de communication et du développement du tourisme côtier.

Pour les professionnels de la forêt, qui ont vécu les grands incendies, faire référence à 1949 suffit à démontrer le danger permanent que court le Massif gascon. Pour ceux qui n'étaient pas nés à cette époque et qui se retrouvent aujourd'hui sylviculteurs, élus locaux, bûcherons ou pompiers forestiers, il devient beaucoup plus difficile après quarante années de maitrise quasi parfaite des incendies par la DFCl, de se persuader de la persistance d'un risque aussi important.

A cet égard, la polémique instaurée au Porge sur le bien-fondé des contre-feux apparaît significative. Pour les pompiers, il n'est pas question de l'utiliser; pour les "civils " impliqués dans la lutte, il s'agit souvent d'un remède incantatoire dans la mesure où peu d'entre eux seraient capables de maîtriser, faute d'expérience antérieure, une technique délicate.

Le succès de la DFCl et du couple "lutte préventive/lutte active", la progressive désertification de la lande, le renouvellement plus ou moins continu des équipes municipales (exemple Le Porge), la confiance placée par tous dans les progrès de la mécanisation, tout concourt à déposséder l'ensemble des intervenants de leur "mémoire collective du feu " et plus encore de leurs réflexes de mobilisation acquis dans les années rouges.

La densification des réseaux de communication, l'augmentation corrélative du trafic routier et surtout le développement estival d'un tourisme côtier de masse, constituent autant de facteurs d'accroissement du risque ${ }^{(2)}$.

L'incendie du Porge restera comme l'illustration parfaite de la nécessité de perpétuer une politique raisonnée d'aménagement de la Côte Aquitaine, préservant les secteurs d'équilibre naturel (SEN) chers à la MIACA. On y a véritablement frôlé la catastrophe notamment à l'intérieur des campings qu'il a fallu évacuer.

Mais, d'autres causes potentielles, à l'influence diffuse mais fondamentale, peuvent encore être évoquées.

II s'agit tout d'abord de la longue stagnation des prix des bois de Pin maritime qui, de 1974 à 1987, sont restés les mêmes en francs courants.

Dans une telle situation, il est quasi miraculeux que la surface forestière du Massit gascon n'ait pas eté amputée (cf. Inventaire forestier national de la Gironde et des Landes de 1988), que la productivité ait continué de croître, il n'est pas douteux que la motivation des professionnels de la filière ne pouvait être à son zénith dans un contexte économique si déprimé.

Parallèlement, les démêlés des associations de $\mathrm{DFCl}$ et des Conseils généraux sur le financement du système de protection contre l'incendie allaient entretenir la division là où la cohésion de tous demeure la condition première du succès.

On ne peut comprendre l'émotion suscitée par les incendies de juillet 1989 parmi les forestiers aquitains qu'en retraçant le contexte conflictuel dans lequel le sinistre survient.

Bien que le département de la Gironde soit peu concerné par les contentieux passés, en cours ou à venir, l'ensemble des sylviculteurs suit avec inquiétude les péripéties qui marquent la réalisation des budgets de la DFCl dans les Landes et le Lot-et-Garonne.

C'est qu'en effet ces départements du Massif gascon sont le théâtre d'un conflit à rebondissements multiples entre Conseil général et Union des Associations de DFCl (Union landaise pour les Landes, Union forestière pour le Lot-et-Garonne).

Sur l'histoire de la création des associations, on se reportera aux deux articles parus dans le numéro spécial de la Revue forestière française de 1975 :

- "La Fédération girondine", par R. Galloy,

- "Les sylviculteurs en face du risque d'incendie", par D. Dorlanne.

(2) Voir, à cet égard, l'étude réalisée par Monsieur Alain Billand "L'efficacité de la défense des forêts contre l'incendie dans le departement des Landes". 


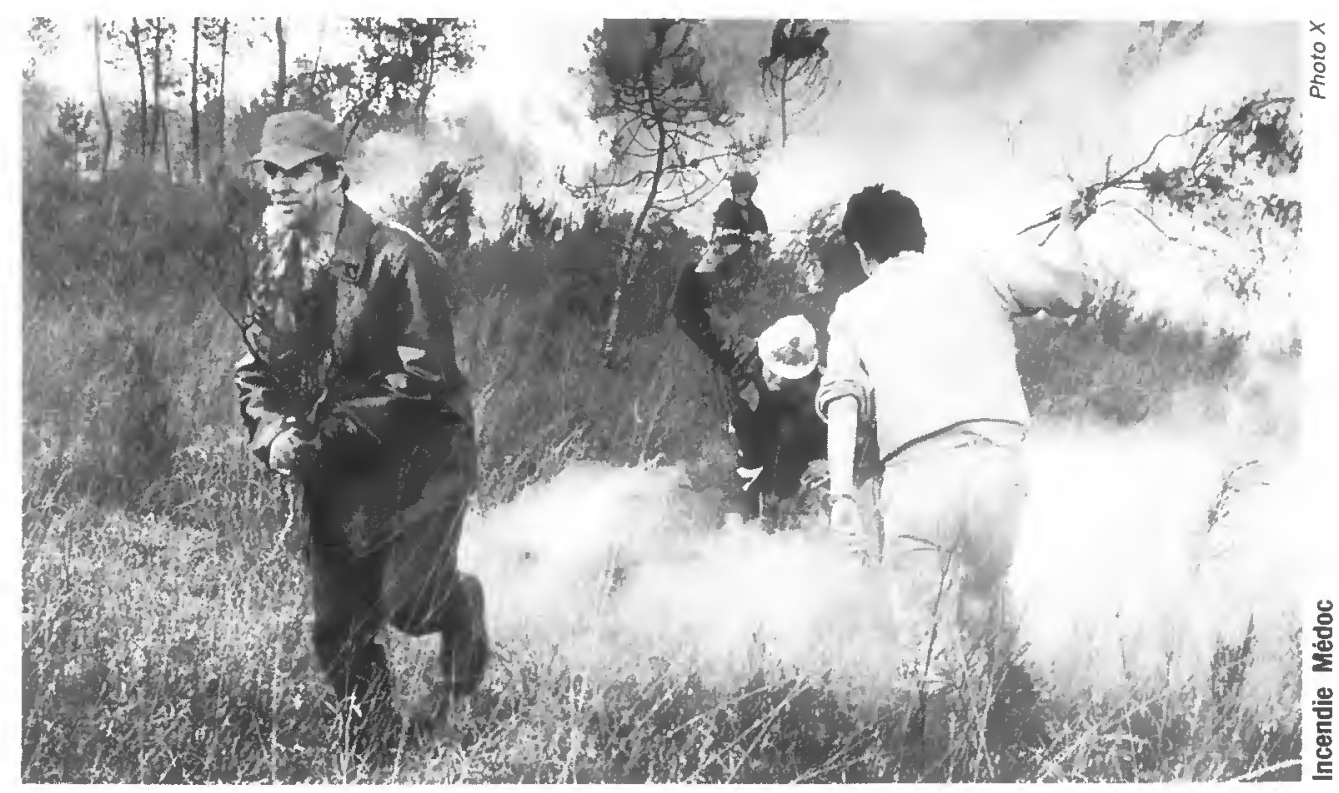

Rappelons avec Marc Gizard (3) que "les associations syndicales autorisées ou constituées d'office sont des groupements de propriétaires - établissements publics ceuvrant dans un but d'utilité privée - sous la tutelle de l'Administration et qui disposent de prérogatives de puissance publique pour exécuter certains travaux d'utilité générale et limitativement énumérés par la loi et pour lever des contributions obligatoires".

Au nombre de 270 , réparties sur les trois départements, elles ont, depuis le décret de 1947, la charge de la prévention qui "cherche, d'une part, à réduire au minimum les causes directes d'incendie et, d'autre part, à organiser l'infrastructure de la forêt, afin que les incendies déclarés prennent le moins d'extension possible, par le compartimentage des massifs, l'équipement en voies de circulation et en points d'eau" (4).

Les problèmes sont apparus en 1974 dans le département des Landes lorsque le Conseil général a prétendu mettre à la charge des associations une partie du financement des dépenses du Corps des sapeurs-pompiers forestiers institué par ce même décret de 1947. On exigeait donc des associations de $\mathrm{DFCl}$ qu'elles participent financièrement à la "lutte active ".

Le Tribunal administratif de Pau, dans une décision du 30 juillet 1975, annulait l'arrêté d'inscription d'office du Préfet en date du 17 mai 1974.

De 1976 à 1980, le problème fut réglé par une convention passée entre I'Union landaise et le Conseil général. Un décret du 5 mai 1980, donnant au Préfet le pouvoir de fixer chaque année la participation des sylviculteurs sur la base "d'une redevance pour service rendu", ralluma le contentieux, les sommes réclamées étant jugées rapidement insupportables ${ }^{(5)}$ par les sylviculteurs.

Le Conseil d'État a annulé, le 18 janvier 1985, le décret de 1980 considérant que "la mission de lutte contre les incendies de forêts confiée aux Corps de sapeurs-pompiers forestiers... est exercée non dans le seul intérêt des propriétaires sylviculteurs... mais dans l'intérêt général de la population; qu'ainsi la contribution imposée par le décret du 5 mai 1980 aux associations syndicales de propriétaires ne correspond pas à la simple rémunération d'un service rendu... et ne saurait être mise à la charge de celles-ci que par la loi".

(3) Marc Gizard: "La fiscalité forestière ", p. 743

(4) R. Galloy.

(5) $1980: 1953700 \mathrm{~F}: 1981: 4084245 \mathrm{~F}: 1982: 4876300 \mathrm{~F}: 1983: 5509080 \mathrm{~F}$. 


\section{Y. LESGOURGUES}

En 1986, nouvelle péripétie où l'on découvre au chapitre "Intérieur et décentralisation " de la loi de Finances un article 75 ainsi rédigé :

"Art. 75 - Les associations syndicales de défense contre les incendies et de remise en valeur de la forêt constituées dans le périmètre défini en exécution de l'article premier de l'ordonnance $n^{\circ}$ 45-582 du 28 avril 1945 sont asujetties à un versement destiné au financement des dépenses du service départemental chargé de la défense des forêts contre l'incendie dans la limite de 20 p. 100 des dépenses de ce service.

Le montant de ce versement est fixé par hectare boisé et peut varier en fonction de la nature de la plantation. Il est arrêté chaque année par délibération du Conseil général après avis d'une commission comprenant notamment des représentants des collectivités locales et des associations syndicales.

Un arrêté interministériel fixe la composition, les modalités de désignation des membres, l'organisation et le fonctionnement de la commission visée à l'alinéa précédent. "

Nouveau contentieux et nouvel arrêt du Conseil d'État (26 mai 1989) qui déclare illégale la Commission départementale qui avait fixé le montant de cette taxe.

Le Lot-et-Garonne ayant suivi l'exemple du Conseil général des Landes, une action se trouve engagée sur les années 1987, 1988 et 1989 contre les arrêtés du Préfet qui fixent le taux de la taxe à $10 \mathrm{~F} / \mathrm{ha}$.

Pour mieux cerner la nature du problème, rappelons que les associations perçoivent des cotisations annuelles de l'ordre de 12 à $17 \mathrm{~F} / \mathrm{ha}$ et qu'un prélèvement égal ou supérieur à $10 \mathrm{~F}$ leur ôte tout moyen de remplir leur tâche de prévention consistant pour l'essentiel en entretien des réseaux existants et création de pistes, points d'eau et pare-feu.

Or, ce travail de prévention, obscur, peu gratifiant, personne ne saurait contester qu'elles l'ont accompli avec une opiniâtreté qui force l'admiration et une efficacité remarquable.

$\dot{A}$ cet égard, les mémoires de A. Billand et I. Pfaff ${ }^{(6)}$, concernant respectivement les associations des Landes et de la Gironde, permettent de mesurer l'ampleur de l'œuvre accomplie.

Depuis 1947, les associations de DFCl du Massif gascon ont réussi à créer et entretenir :

\begin{tabular}{|c|c|c|c|}
\hline & Pistes & Fossés & Points d'eau \\
\hline Gironde & $3530 \mathrm{~km}$ & $8000 \mathrm{~km}$ & 686 \\
\hline Landes $\ldots \ldots \ldots \ldots \ldots \ldots \ldots \ldots$ & $8000 \mathrm{~km}$ & $12000 \mathrm{~km}$ & 231 \\
\hline Lot-et-Garonne $\ldots \ldots \ldots \ldots \ldots \ldots$ & $2400 \mathrm{~km}$ & $3500 \mathrm{~km}$ & 22 \\
\hline
\end{tabular}

Sans vouloir établir de hiérarchie entre ces causes profondes, il paraît clair que leur juxtaposition constitue un facteur aggravant du risque qui se concrétise pour la première fois au Porge en juillet 1989 .

Mais ce sont à l'évidence des conditions climatiques exceptionnelles qui entraîneront le déclenchement de cet incendie "pas comme les autres".

C'est qu'en effet l'année 1989, si l'on excepte le mois d'avril, demeurera comme une année de grande sécheresse. II ne pleuvra que $678 \mathrm{~mm}$ en 1989 contre $935 \mathrm{~mm}$ en 1988. Cinq cents points d'eau jugés permanents par les pompiers devront être déclassés.

Par ailleurs, les dysfonctionnements multiples constatés auront un rôle majeur : hésitation dans le commandement, insuffisance dans la garde du feu au soir du premier jour, faillite du système de transmission, absence de coordination entre moyens terrestres et moyens aériens, enfin graves dissensions entre locaux et professionnels du feu.

Mais, au-delà des polémiques souvent inutiles parce que trop tardives, il apparaît souhaitable de tirer les principaux enseignements de ce feu heureusement exceptionnel et de formuler quelques propositions.

(6) cf. ètudes citees en bibliographie page 71 . 


\section{CONCLUSIONS PROVISOIRES - PROPOSITIONS}

Les conclusions à tirer de cet événement sont multiples.

- Le Massif gascon, premier massif forestier de la CEE, apparaît à nouveau comme vulnérable aux grands incendies.

- La forêt est de plus en plus revendiquée comme un patrimoine commun à tous les Aquitains. Ceci doit aider à la résolution de la crise du système $\mathrm{DFCl}$ évoquée précédemment, mais exige en retour un effort d'explication permanent de la part des professionnels en direction de nouveaux intervenants.

- Pour la première fois, l'engagement de moyens aériens de type "Canadair " est apparu nécessaire dans le Massif gascon.

- Une inquiétante coupure entre pompiers professionnels et forestiers locaux s'est manifestee pendant les événements. Cette incompréhension ne laisse pas d'inquiéter.

- Le système de cloisonnement en vigueur sur les zones dunaires de la forêt publique ne facilite pas la lutte contre le feu, car ne tenant pas suffisamment compte du relief.

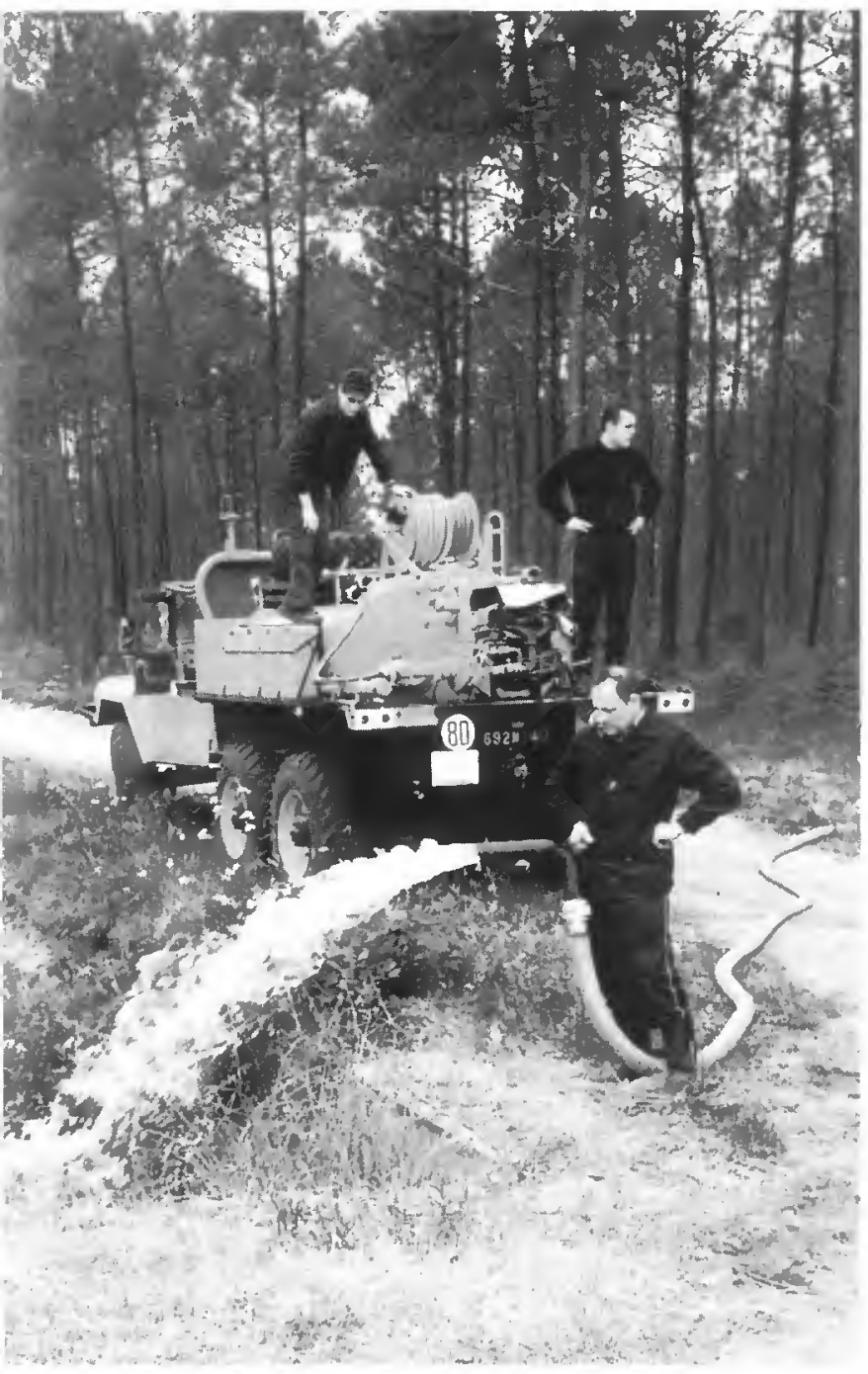

- Le ravitaillement des nombreux camions engagés dans un feu de grande ampleur nécessite de nombreux points d'eau aménagés et répertoriés.

Installation mobile alimentée par forage en forêt (Union landaise)

Photo CPFA

Les principales propositions que l'on peut formuler sont les suivantes:

\section{- Sortir au plus tôt du conflit de la DFCl dans les Landes et en Gironde}

Les derniers développements en date, et notamment la décision de mise en sommeil des associations à la suite du vote de la loi de Finances pour 1989 portant à $12 \mathrm{~F} /$ ha la participation des sylviculteurs au financement de la $\mathrm{DFCl}$, ne laissent rien présager de bon. Ainsi a-t-on assisté à la première manifestation de l'histoire de la sylviculture, le 17 février 1990, dans les rues de la Préfecture des Landes où plus de 1000 sylviculteurs s'ètaient rassemblés.

- Relancer la vie associative, base de la DFCl et ciment social de la vie du Massif gascon

Pour ce faire, il convient de réfléchir à la possibilité de faire travailler en commun toutes les parties concernées au sein par 
exemple de comités départementaux "Feux de Forêts". La professionnalisation des sapeurspompiers, la pluriactivité de plus en plus généralisée des sylviculteurs font que les deux protagonistes se connaissent de moins en moins bien. Or, le système ne peut fonctionner tant en matière de prévention que de lutte active, que si les parties travaillent en harmonie et échangent leur savoir-faire respectif.

\section{- Réfléchir à la pérennisation de moyens aériens sur le Massif}

Le stationnement de deux Canadair sur la base de Cazaux a été promis par le ministre de l'Intérieur à la suite de l'incendie. Qu'en est-il aujourd'hui ?

Par ailleurs, les pompiers souhaiteraient disposer d'hélicoptères bombardiers d'eau aptes à combattre des feux naissants.

C'est donc toute la conception de l'articulation moyens terrestres/moyens aériens qui est à revoir.

- Étendre le système de points d'eau permanents mis au point dans le département des Landes à l'ensemble du Massif

Les sources traditionnelles d'approvisionnement (châteaux d'eau forestiers, réserves au sol de $60 \mathrm{~m}^{3}$, points d'eau naturels ou aménagès) sont insuffisantes. Dans le département des Landes, on a recensé, avant la mise en ceuvre du programme de forages, une moyenne de 3200 ha par point d'eau alors que l'optimum requis paraît de 500 ha pour un point d'eau.

L'Union landaise, avec l'aide de la Communauté européenne et du FFN, s'est lancẻe dans un programme coordonné de création de 600 points d'eau permanents à partir de forages réalisés dans la nappe phréatique. Les pompiers se sont équipés de pompes mobiles et autonomes montées sur remorque ou chassis du véhicule, que l'on descend dans le forage en trois minutes. On peut ainsi remplir quinze camions en une heure.

- Instaurer une coopération interdépartementale ou régionale sur les problèmes de $\mathrm{DFCl}$

Il n'est plus possible d'ignorer la communauté d'intèrêts qui lie les départements forestiers aquitains sur le plan de la DFCl. L'absence de structure interdépartementale, le refus, jusqu'à l'heure, de la Région de participer à des actions DFCl constituent des anomalies dirimantes.

- Intégrer les corps de sapeurs-pompiers forestiers dans les SDIS

Cette mesure éviterait les doubles emplois, les confusions multiples, voire les rivalités et aurait le mérite de la clarté.

Sur ce point, les conclusions du rapport du ministère de l'Intérieur (mai 1985) mériteraient d'être suivies: " I me semble préférable de s'en tenir à un seul établissement public départemental intégrant les sapeurs-pompiers forestiers pour lequel la défense de la forêt contre l'incendie constituerait une mission de droit commun".

\section{CONCLUSIONS}

L'incendie de Lacanau-Le-Porge peut être analysé de multiples façons.

Certes, dans l'ensemble des 60000 ha brûlés en 1989 dans l'hexagone, il peut paraître relativement marginal.

Pour les Aquitains, au contraire, il mérite une atłention toute particulière. Il a réactivé un ensemble de réflexes qui tendaient à s'émousser après quarante années de succès relatif.

Outre la (re)prise de conscience de la possibilité de survenue d'un sinistre de type 1949, l'incendie aura permis de situer les enjeux à leur véritable hauteur.

La forêt landaise, forêt artificielle constituée à partir d'une essence autochtone, a su générer une intense activité économique qui la met aux tout premiers rangs des massifs forestiers européens.

L'exceptionnelle efficacité de son système $\mathrm{DFCl}$, le dynamisme des organisations professionnelles de sylviculteurs, la richesse du tissu industriel ont conduit les décideurs de tous bords à 
considérer que le massif jouissait d'une relative prospérité ne nécessitant pas de grandes mesures d'accompagnement. C'est, par ailleurs, un trait dominant "du mal français " que de ne savoir pas valoriser ses atouts et de préférer le saupoudrage aux arbitrages.

Ainsi, a-t-on laissé se dégrader un système basé sur la responsabilisation des sylviculteurs aux travers d'associations pour lesquelles le législateur avait judicieusement prévu qu'elles sauraient s'auto-taxer.

Ainsi, n'a-t-on pas vu qu'une situation de prix des bois totalement étale de 1974 à 1987 ne laissait rien présager de bon pour l'entretien d'un massif aussi essentiel à l'économie forestière française.

Aussi, n'a-t-on pas compris les redoutables conséquences du cycle climatique exceptionnel inauguré par le froid de janvier 1985 faisant disparaître 30000 ha de pins et générant des attaques parasitaires multiples.

Les trente mois consécutifs de sécheresse que nous venons de vivre, l'ampleur exceptionnelle de l'attaque de chenilles processionnaires en cours (200 000 ha touchés), l'abaissement dramatique des nappes phréatiques et le tarissement de nombreux points d'eau, montrent à l'évidence que l'Aquitaine se prépare à vivre "l'année de tous les dangers".

Puissent les leçons des incendies de 1989 être tirées afin de repenser la distribution des moyens qui permettrait de résoudre au plus tôt le lancinant conflit DFCl/Conseil général ${ }^{(7)}$ et de relancer une complémentarité harmonieuse entre sylviculteurs et pompiers forestiers.

Puissent les Aquitains et ceux qui les visitent mesurer l'ampleur du travail accompli depuis un siècle et demi pour valoriser les sols squelettiques des Landes, la formidable ressource naturelle accumulée et l'importance écologique et économique de celle-ci.

\author{
Y. LESGOURGUES \\ Directeur du \\ CENTRE DE PRODUCTIVITÉ \\ ET D'ACTION FORESTIÈRE D'AQUITAINE \\ Maison de la Forêt \\ 17, rue Esprit des Lois \\ 33080 BORDEAUX CEDEX
}

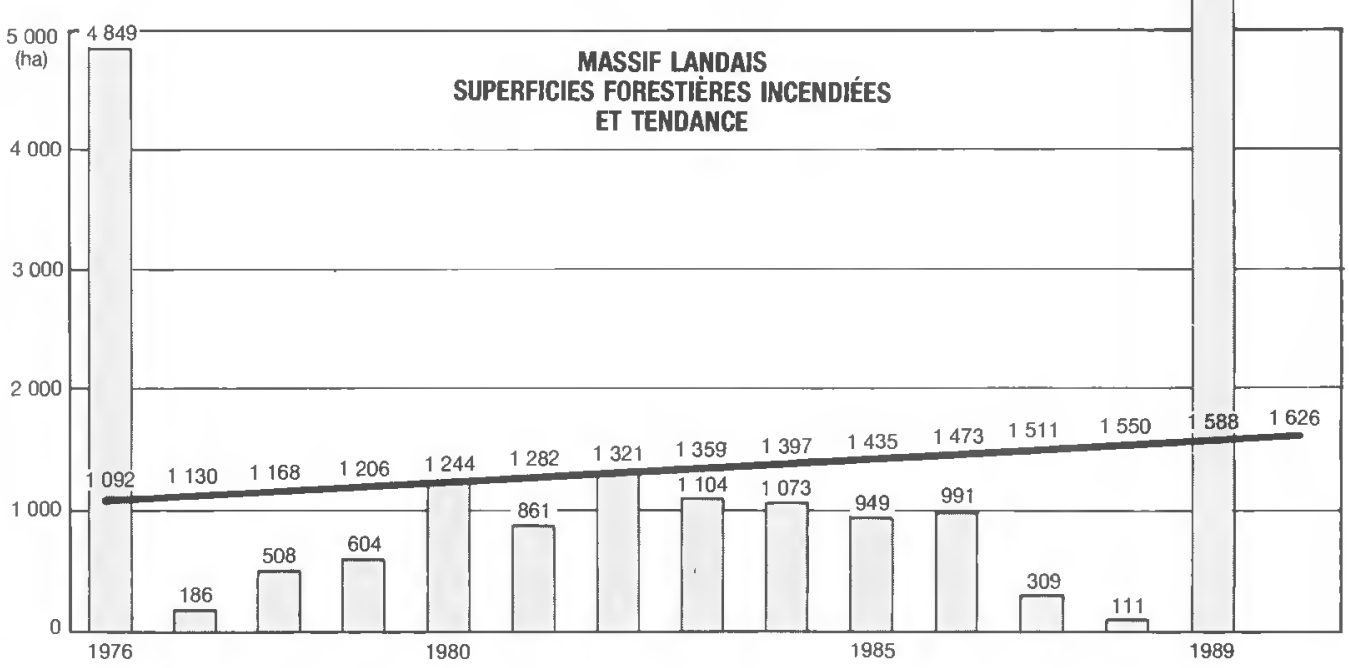

(7) Rappelons que les sommes en jeu sont de l'ordre de 10 à 20 millions de francs, alors que le montant de la lutte contre les incendies de forêts dans le Sud-Est est estimée à 1 milliard de francs par an... 


\section{LES INCENDIES DU 31 MARS 1990 EN GIRONDE}

Dans la nuit du 31 mars au $1^{\mathrm{er}}$ avril 1990, dans des conditions de sécheresse exceptionnelles, le feu démarrait de la commune de Saint-Aubin (Gironde), aux portes de Bordeaux, à cinq endroits différents; poussé par un vent violent et tourbillonnant, il allait rapidement prendre de l'extension, s'étendre vers l'ouest sur $25 \mathrm{~km}$ pour être maîtrisé aux portes même de Carcans. Dans le même temps, un autre incendie de moindre importance se développait sur Le Barp.

\section{LES PREMIĖRES RÉACTIONS}

Dès le dimanche, le chiffre de 5000 ha brûlés circulait dans les rédactions. Les premiers communiqués faisaient état de l'importance des forces DFCl engagées dans la bataille. Les Canadair, stationnés à Marignane (et non à Cazaux, comme le pensaient certains), ne seraient à même d'intervenir que le dimanche.

Le mardi 3 avril, le maire de Sainte-Hélène, dans une conférence de presse musclée, mettait en cause le fonctionnement du Corps de pompiers ef la tactique au feu. II trouve à ses côtés les Présidents Martres et Larrouy ainsi que les maires sinistrés qui se montraient très critiques quant à l'absence de liaison une fois de plus observée entre professionnels du feu et professionnels de la forêt.

Écoutons Monsieur Yves Lecaudey, maire de Sainte-Hélène: " depuis dimanche, j'entends les autorités multiplier les communiqués de victoire, transformer un désastre en triomphe, se masquer la face par leurs plus criantes insuffisances. En octobre, nous avons remis un rapport de synthèse tirant toutes les conclusions de l'incendie du Porge et faisant un ensemble de propositions urgentes. Aujourd"hui, alors que rien n'a été fait, nous pouvons dire: même constat, mêmes causes, mêmes effets. Un seul élément nouveau: nous assistons à la providentielle polémique sur les Canadair, sujet qui camoufle le problème de fond. À savoir que nos procédures de lutte sont à revoir de fond en comble. C'est un problème de hiérarchie, un problème de responsabilité, un problème de mise en couvre; c'est un problème d'hommes."

Dans le Médoc, littéralement abasourdi par l'enchaînement de deux sinistres aussi importants à huit mois d'intervalle, la polémique va bon train, surtout lorsque l'on constate que les directives ont été de sauvegarder coûte que coûte les habitations (nombreuses dans cette zone proche de Bordeaux), au détriment des futaies de Pin maritime. 


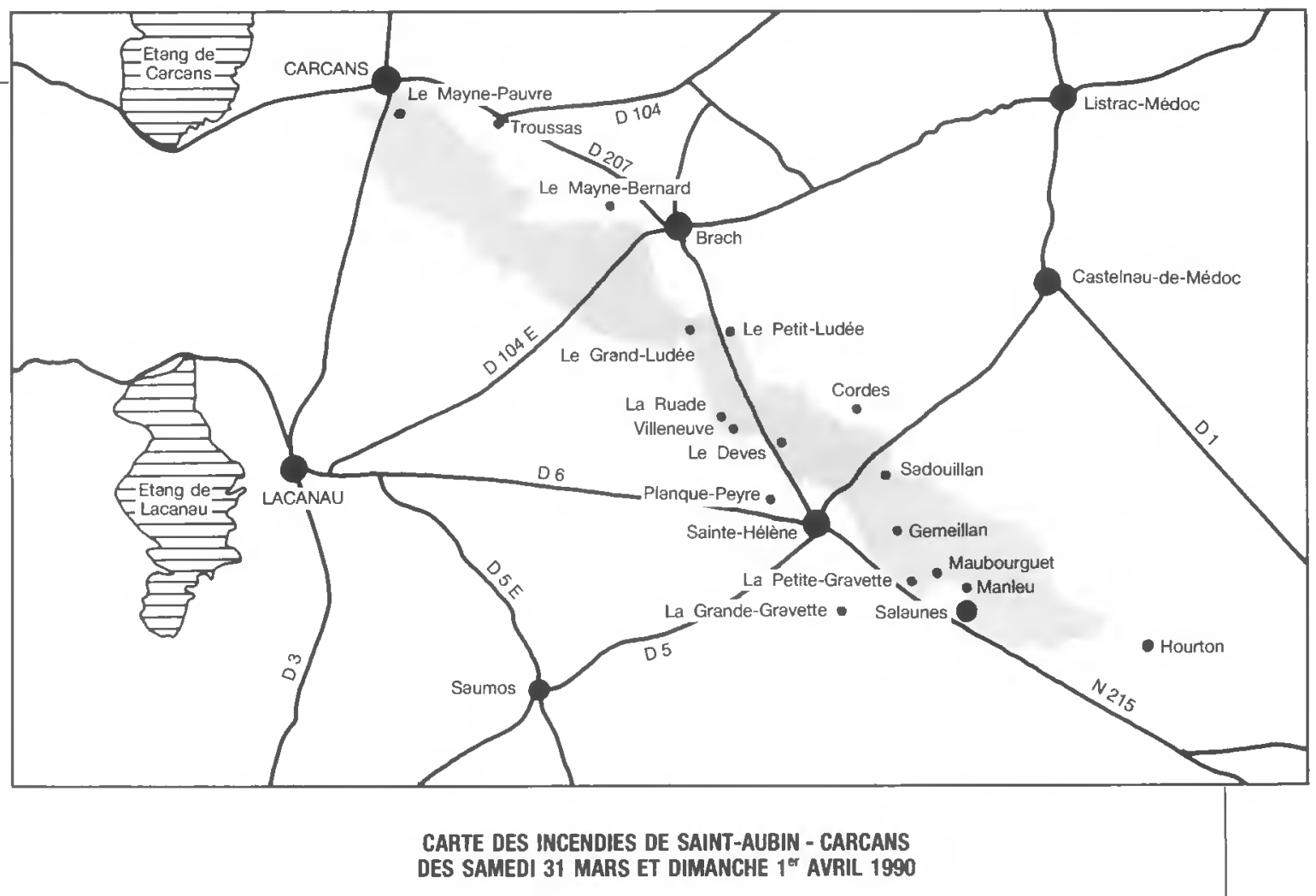

BILAN

Avec plus de 5600 ha brûlés, l'incendie du $1^{\text {er }}$ avril 1990 surpasse de très loin celui du Porge-Lacanau de triste mémoire.

En l'espace de huit mois, près de 10000 ha de forêt de prơuction auront été rayés de la carte par l'incendie.

La répartition par commune s'analyse comme suit:

\begin{tabular}{|c|c|}
\hline \multirow[t]{2}{*}{ 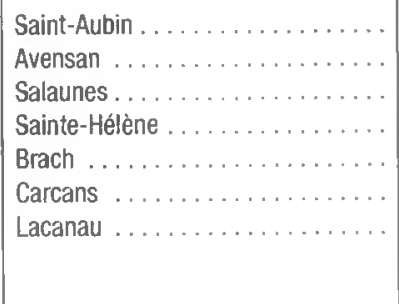 } & $\begin{array}{r}180 \text { ha } \\
34 \text { ha } \\
1198 \text { ha } \\
2185 \text { ha } \\
849 \text { ha } \\
1166 \text { ha } \\
24 \text { ha }\end{array}$ \\
\hline & 5636 ha \\
\hline
\end{tabular}

Par ailleurs, les estimations en volume des bois à exploiter s'établissent à $700000 \mathrm{~m}^{3}$ dont la moitié en gros bois. 


\section{CONCLUSIONS}

Que dire après ce nouveau coup dur pour la forêt aquitaine?

Qu'il est temps, plus que temps de redonner toute sa cohérence (d'où découlera l'efficacité) au système de DFCl Iandais.

La relative similitude des analyses professées successivement par le Président du Conseil général de la Gironde, le Président du Syndicat des Sylviculteurs du Sud-Ouest et plus récemment le ministère de l'Agriculture constitue peut-être un signe encourageant.

"La forêt de 1990 n'est plus celle des annèes 50. Le contexte économique de la sylviculture, le tourisme estival de masse, l'urbanisation et l'occupation humaine de la forêt conduisent à modifier radicalement notre approche du problème. Le feu, c'est finalement l'échec de la prévention».

Philippe Madrelle

Président du Conseil général

4 mai 1990

"Nous demandons simplement que soit pris en compte le risque social déterminant qui veut que la forêt attire les citadins et les touristes, qu'elle soit de plus en plus considérée comme un bien commun dont on peut user à sa guise. La forêt de Gascogne a toujours été ouverte et, de ce point de vue, la plus vulnérable."

...

"Alors quel est le choix? Soit l'Etat pense que nous devons payer une partie de la lutte active, et alors nous abandonnons la prévention, soit l'État pense que nous devons payer la prévention et que c'est suffisant.

Mais nous ne paierons pas et la prévention et la lutte active *.

Jean-Louis Martres

Président du Syndicat

des Sylviculteurs du Sud-0uest

17 février 1990

"La sécheresse de l'année 1990, qui fait suite à celles de 1988 et 1989, provoque une situation exceptionnelle caractérisée par des risques inhabituels pour le Massif landais...

... Les difficultés rencontrées pour le financement des actions de prévention avec la mise en sommeil des associations syndicales de DFCl, sont de nature à aggraver les difficultés malgré l'accroissement des moyens d'intervention et l'amélioration de la coordination de la lutte décidée récemment par le ministère de l'Intérieur "(1)."

DERF

Dossier de presse sur la prévention

28 mai 1990

Et puisqu'aujourd'hui, aucune décision ne saurait être prise qu'argumentée en termes de retombées économiques, empruntons au même dossier de la DERF cette conclusion qui situe une partie des enjeux:

"Au total, la filière forêt-bois génère 40000 emplois dans la région Aquitaine.

À la différence de la forêt du Sud-Est, la protection de la forêt landaise est nécessaire, non seulement pour sauvegarder un patrimoine naturel, mais aussi pour préserver une activité économique qui est la première pour son importance au plan régional».

(1) Rappelons que les mesures annoncees depuis l'incendie du $1^{\text {er }}$ avril sont:

- location de deux hélicoptères bombardiers d'eau et d'un avion de type Macavia:

- mise en place d'une Unité d'Instruction et d'Intervention de la Sécurité civile regroupant une centaine d'appelés du contingent;

- création d'un Centre interrégional de Commandement opérationnel de la Sécurité civile :

- fusion des commandements du Service départemental d'Incendie de la Gironde et du Corps des sapeurs-pompiers forestiers. 


\section{BIBLIOGRAPHIE}

BILLAND (A.). - L'Efficacité de la DFCl dans le département des Landes. - Mémoire ENITEF, 1985.

Les Incendies de forêts. - Revue forestière française, $n^{\circ}$ spècial 1975, tome 2 (1974: tome 1).

Incendies. - Journal Sud-Ouest, numéro spécial, juillet 1989, $28 \mathrm{p}$.

LESGOURGuES (Y.), ROUX (L.), DE SEZE (P.). - Le Porge-Lacanau. Caracterisation des dégåts, Évaluations des préjudices. Approche des coûts de reconstitution. - Bordeaux : CPFA, septembre 1989.

MANARILLO (J.). - La Crise du système landais de protection contre l'incendie. - DESS Administration locale, 1986.

MINISTĖRE DE L'INTĖRIEUR ET DE LA DÉCENTRALISATION, MINISTĖRE DE L'AGRICULTURE. — Les Problèmes et les perspectives de la défense de la forêt contre l'incendie dans les dèpartements des landes de Gascogne. - Rapport, mai 1985.

OFFICE NATIONAL DES FORÊTS. - L'Incendie de forêts sur Le Porge-Lacanau. Constatations provisoires. Bordeaux : Direction régionale ONF.

PFAFF (Isabelle). - Organisation et coût de la DFCl en Gironde. Bilan et perspectives. - Mémoire ENITEF, 1983.

PINAUDEAU (C.). - Politique forestière en matière d'incendie: l'impossible prevention. - Université Bordeaux I - Centre d'Analyse politique comparée. 